\title{
Chrysotile Asbestos
}

National Cancer Institute

\section{Source}

National Cancer Institute. Chrysotile Asbestos. NCI Thesaurus. Code C45529.

A naturally occurring, fibrous and silky, serpentine asbestos mineral with a color rang ing from gray-white to yellow-green and is the most abundant and widely-used form of asbestos. Chrysotile is widely used in industry for various purposes. Chronic inhalation of its dust may cause asbestosis and increases the risk of digestive tract cancer, laryngeal cancer, mesothelioma and other lung cancers. Chrysotile asbestos is a known human carcinogen. $(\mathrm{NCl05})$ 